\title{
A Case Study of Combined Perception-Based and Perception-Free Spinal Cord Stimulator Therapy for the Management of Persistent Pain after a Total Knee Arthroplasty
}

\author{
Ivan Urits (D) - Mohamed Osman • Vwaire Orhurhu • Omar Viswanath • \\ Alan D. Kaye · Thomas Simopoulos · Cyrus Yazdi
}

Received: April 17, 2019 / Published online: August 20, 2019

(C) The Author(s) 2019

\begin{abstract}
Introduction: Total knee arthroplasty (TKA) is an effective treatment modality for severe osteoarthritis of the knee. Causes of pain following TKA are poorly understood; however, patient-specific biology and various neuropathic underlying mechanisms such as neuroma formation and complex regional pain
\end{abstract}

Enhanced digital features To view enhanced digital features for this article go to https://doi.org/10.6084/ m9.figshare.9199763.

I. Urits $(\bowtie) \cdot$ M. Osman · V. Orhurhu .

T. Simopoulos · C. Yazdi

Department of Anesthesia, Critical Care, and Pain

Medicine, Beth Israel Deaconess Medical Center,

Harvard Medical School, Boston, MA, USA

e-mail: iurits@bidmc.harvard.edu

O. Viswanath

Valley Anesthesiology and Pain Consultants,

Envision Physician Services, Phoenix, AZ, USA

O. Viswanath

Department of Anesthesiology, University of

Arizona College of Medicine, Phoenix, Phoenix, AZ, USA

O. Viswanath

Department of Anesthesiology, Creighton

University School of Medicine, Omaha, NE, USA

A. D. Kaye

Department of Anesthesiology, Louisiana State

University Health Sciences Center, New Orleans, LA, USA syndrome (CRPS) have been suggested. Our case demonstrated the successful treatment of CRPS in the knee with the use of combination therapy in spinal cord stimulator.

Case: We present a 71-year-old Caucasian nonHispanic male who presented with chronic left knee pain after undergoing a total knee arthroplasty (TKA) 18 months prior. Following his TKA, he reported doing well in the acute postoperative period but began to develop progressively worsening left knee pain at approximately the third post-operative week. He underwent a successful spinal cord stimulator (SCS) trial and subsequent implantation of two 16-contact Boston Scientific leads with a Boston Scientific Spectra WaveWriterTM SCS system. Upon first post-procedural follow-up, and moreover at his 6-month follow-up, the patient reported complete resolution of his symptoms. Discussion: The development of persistent pain following TKA is a significant complication that is often challenging to treat. Our case demonstrated the successful treatment of CRPS in the knee with the use of combination therapy in spinal cord stimulator therapy. We anticipate that more data will continue to emerge to assess for the safety and efficacy of combination therapy.

Keywords: Combination therapy; Complex regional pain syndrome; Neuromodulation; Spinal cord stimulator; Total knee arthroplasty 


\section{INTRODUCTION}

Total knee arthroplasty (TKA) is an effective treatment modality for severe osteoarthritis of the knee. Though eventual mechanical failure may be anticipated with long-term activity and normal use, as many as $28 \%$ of patients who undergo an uncomplicated TKA and postoperative course may experience persistent moderate to severe knee pain [1,2]. Causes of pain following TKA are poorly understood; however, patient-specific biology and various neuropathic underlying mechanisms such as neuroma formation and complex regional pain syndrome (CRPS) have been suggested [1, 3-5]. Informed consent was obtained for the publication of this case. The aim of this case study is to present the use of combination spinal cord stimulator (SCS) therapy for the management of persistent pain following TKA.

\section{CASE REPORT}

Our patient was a 71-year-old Caucasian nonHispanic male who presented with chronic left knee pain after undergoing a TKA 18 months prior. He had an uncomplicated past medical history of hypertension, hyperlipidemia, and reflux, which were well controlled with medical management. He was 70 inches tall and weighed $215 \mathrm{lbs}$. He had no history of any additional surgeries. He was married and lived at home with his wife. He was now retired and prior to his surgery maintained an active lifestyle including exercise and daily life activities. His social history included social alcohol consumption and was negative for tobacco use (Fig. 1).

Following his TKA, the patient complained of persistent and progressively worsening postoperative pain of his knee. Orthopedic evaluation determined his implant to be normally functioning without evidence of hardware loosening or infection. On presentation, he described the pain as sharp, 7/10 (VAS) in severity though poorly defined, with radiation to the left ankle, and associated paresthesias in the same distribution. On physical exam, he experienced symptoms of hyperesthesia and

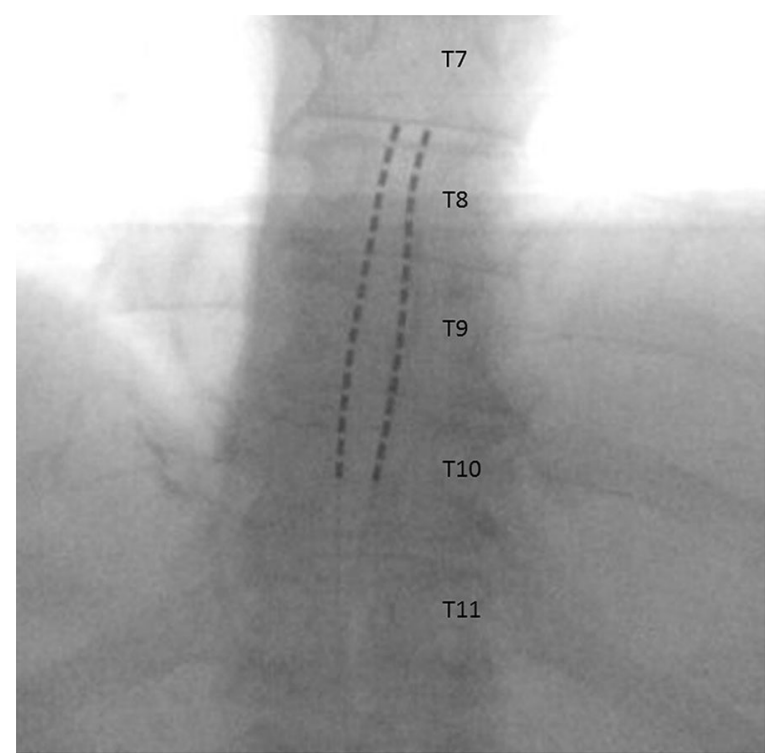

Fig. 1 Anterior-posterior radiographic film demonstrating permanently implanted leads positioned at the top of the T8 vertebral body and spanning vertebra T8-T10

allodynia along the anterior aspect of his knee. His postsurgical incision was well healed, without evidence of erythema, swelling, or gross changes in color. Strength was 5/5 with flexion and extension of the lower left leg, but was limited by guarding due to pain. His right lower extremity had 5/5 strength in all myotomes, and otherwise he had 5/5 strength in the left leg with flexion and extension of the thigh, inversion, eversion, plantar flexion, and dorsiflexion of his left foot.

Given his symptomatology of hyperesthesia, allodynia, preserved strength, and absence of orthopedic aberrancy, a diagnosis of postsurgical CRPS of the left lower extremity was made. Initial management of his pain was conservative including physical therapy, non-steroidal anti-inflammatory, muscle relaxant, neuropathic, and opioid medications, which provided little or no relief of symptoms. Following a trial of lumbar sympathetic block, he reported improvement in pain, with a reduction on VAS to $3 / 10$, lasting approximately 1 month in duration. Given a dramatic improvement in his knee symptoms, he subsequently underwent a successful SCS trial and uneventful implantation of two 16-contact Boston Scientific leads 
with a Boston Scientific Spectra WaveWriter ${ }^{\mathrm{TM}}$ SCS system. His generator was programmed to provide simultaneous traditional paresthesiabased therapy at a frequency of $50 \mathrm{~Hz}$ along with the combination of burst therapy at a frequency of $450 \mathrm{~Hz}$, with six pulses per burst, as well as sub perception stimulation therapy at a frequency of $1.2 \mathrm{kHz}$. Upon first post-procedural follow-up, the patient experienced dramatic pain relief, reporting complete resolution of his symptoms. Moreover, at 6 months post SCS implantation, he continued to experience complete resolution and had successfully completed an opioid wean to discontinuation.

\section{DISCUSSION}

The development of persistent pain following TKA is a significant complication that is often challenging to treat. In the absence of mechanical pathology, surgical revision for poorly defined persistent chronic pain leads to worsened outcomes [3]. Instead, a conservative approach has been demonstrated to have some benefit; in a long-term study of 18 patients who had chronic unexplained pain following surgery, 55\% reported improvement with reassurance and watchful waiting at 5-year follow-up [6]. Still, few therapeutic options are available to provide relief in this challenging patient population. Our case suggests that combination therapy in SCS may be an effective modality to treat CRPS in the lower extremity. Combination SCS therapy includes both paresthesia and highfrequency sub-perception-based therapies, the combination of which is customizable to improve patient pain relief. North et al., in the WHISPER study, demonstrated supporting evidence for sub-perception therapies in combination with supra-perception therapies. They found an increased $62 \%$ patient responder rate when the combination therapy was implemented in patients with chronic pain [7]. We anticipate more data will continue to emerge and further provide supporting evidence for the safety and efficacy of combination therapy when compared to either paresthesia or sub perception-based therapies individually.

\section{CONCLUSIONS}

Persistent pain following TKA is an important complication and is often difficult to treat. Combination SCS may be an effective modality for the treatment of persistent pain in the lower extremity following TKA; further studies are needed to evaluate the long-term efficacy.

\section{ACKNOWLEDGEMENTS}

We thank the participant of the study.

Funding. No funding or sponsorship was received for this study or publication of this article.

Authorship. All named authors meet the International Committee of Medical Journal Editors (ICMJE) criteria for authorship for this article, take responsibility for the integrity of the work as a whole, and have given their approval for this version to be published.

Disclosures. There are no grants, sponsors, funding sources, or direct financial support to disclose. Ivan Urits, Mohamed Osman, Vwaire Orhurhu, Omar Viswanath, Alan D. Kaye, Thomas Simopoulos, and Cyrus Yazdi have nothing to disclose.

Compliance with Ethics Guidelines. Informed consent was obtained for the publication of this case.

Data Availability. Data sharing is not applicable to this article as no datasets were generated or analyzed during the current study.

Open Access. This article is distributed under the terms of the Creative Commons Attribution-NonCommercial 4.0 International License (http://creativecommons.org/licenses/ by-nc/4.0/), which permits any noncommercial use, distribution, and reproduction in any medium, provided you give appropriate credit to the original author(s) and the source, provide a link to the Creative Commons license, and indicate if changes were made. 


\section{REFERENCES}

1. Hasegawa M, Tone S, Naito Y, Wakabayashi H, Sudo A. Prevalence of Persistent Pain after Total Knee Arthroplasty and the Impact of Neuropathic Pain. J Knee Surg. 2018; (Epub ahead of print).

2. Beswick AD, Wylde V, Gooberman-Hill R, Blom A, Dieppe P. What proportion of patients report longterm pain after total hip or knee replacement for osteoarthritis? A systematic review of prospective studies in unselected patients. BMJ Open. 2012;2(1):e000435.

3. Wylde V, Beswick A, Bruce J, Blom A, Howells N, Gooberman-Hill R. Chronic pain after total knee arthroplasty. EFORT Open Rev. 2018;3(8):461-70.
4. Phillips JR, Hopwood B, Stroud R, Dieppe PA, Toms AD. The characterisation of unexplained pain after knee replacement. Br J Pain. 2017;11(4):203-9.

5. Preston S, Petrera M, Kim C, Zywiel MG, Gandhi R. Towards an understanding of the painful total knee: what is the role of patient biology? Curr Rev Musculoskelet Med. 2016;9(4):388-95.

6. Elson DW, Brenkel IJ. A conservative approach is feasible in unexplained pain after knee replacement. J Bone Joint Surg Br. 2007;89-B(8):1042-5.

7. North J, WHISPER Study Group, Chen L, Jain R. WHISPER: a multicenter, prospective randomized controlled trial evaluating subperception SCS| 2018 world congress on regional anesthesia and pain medicine. In: World Congress on Regional Anesthesia and Pain Medicine. New York; 2018; p. 4926. 